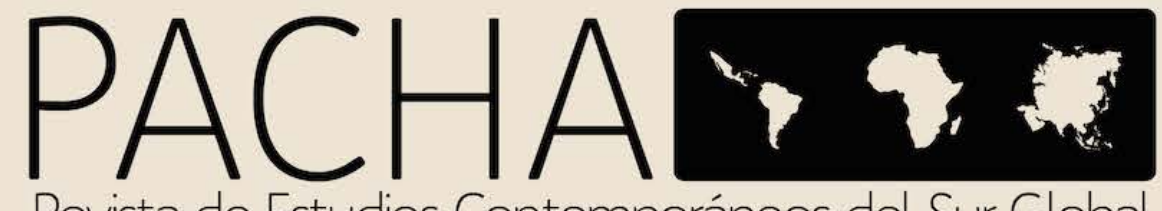

Revista de Estudios Contemporáneos del Sur Global Joumal of Contemporary Studies of the Global South Revista de Estudos Contemporâneos do Sul Global 


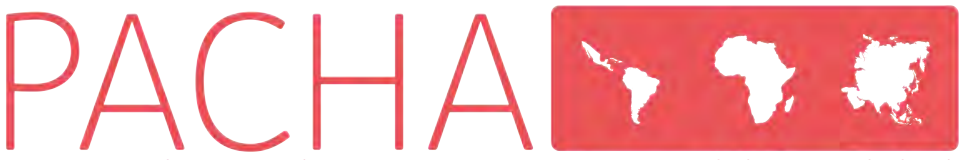

Revista de Estudios Contemporáneos del Sur Global

Joumal of Contemporary Studies of the Global South

\section{Equipo Editorial}

Carolina Díaz R.

Directora Editorial

CICSH-AL Centro de Investigaciones en Ciencias Sociales y Humanidades desde América Latina, Ecuador revistapacha@religacion.com; carolinadiaz@religacion.com

Roberto Simbaña Q.

Coordinador Editorial

CICSH-AL Centro de Investigaciones en Ciencias Sociales y Humanidades desde América Latina, Ecuador robertosimbana@religacion.com

\section{Editores Asociados}

Paola Andrea Tovar. Universidad De Montreal, Colombia. Editora Asociada en Antropología

Mitchell Alberto Alarcón Díaz. Universidad Nacional Mayor de San Marcos, Perú. Editor Asociado en Educación

Marcela Cristina Quinteros. Universidade Estadual de Maringá, Brasil. Editora Asociada en Historia Latinoamericana

Aboutaleb Sedatee Shamir. Islamic Azad University, Iran. Editor Asociado en Educación y Medio Oriente

Mateus Gamba Torres. Universidade de Brasilia - UNB, Brasil. Editor Asociado en Historia Mirna Yazmin Estrella Vega. Universidad Nacional Autónoma de México, México. Editora Asociada en Sociología

Rodrigo Navarrete Saavedra. Universidad Aus tral de Chile, Chile. Editor Asociado en Ciencias Políticas

Aygul Zufarovna Ibatova. Tyumen Industria University, Rusia. Editora Asociada en Humahidades sobre Asia
Shokhrud Fayziev Farmanovich. Tadqiqot, Tashkent, Uzbekistan. Editor Asociado en Desarrollo sobre la Comunidad de Estados Independientes, Asia.

Mª Aránzazu Serantes López. Woolf University, España. Editora Asociada en Humanidades digitales

Fabricio Espinosa Ortiz. Consejo Nacional de Ciencia y Tecnología, México. Editor Asociado en Geografía Humana

Marcelo Starcenbaum. Universidad Nacional de La Plata, Argentina. Editor Asociado en Filosofía e Historiografía

Siti Mistima Maat. Universiti Kebangsaan Malaysia, Malaysia. Editora Asociada en Innovación de aprendizaje

Carla Vanessa Zapata Toapanta. Universidad de Salamanca, España. Editora Asociada en Latinoamericanismo

\section{Consejo Editorial}

Andrea Paola Cantarelli, Universidad Nacional de Cuyo, Argentina
Federico Cabrera, Universidad Nacional de San Juan/CONICET, Argentina

Gamaniel David Suárez Cobix, Universidad Veracruzana, México

Gloria Concepción Tenorio Sepúlveda, Tecnológico de Estudios Superiores de Chalco, México

Héctor García Cornejo, Universidad Michoacana de San Nicolás de Hidalgo, México

Jorge Gilberto Bonilla Macas, Universidad Católica de Cuenca, Ecuador

José Alexander Rubiano Pedroza, Universidad de Pamplona, Colombia

María Dolores Sancho, Universidad Nacional del Comahue, Argentina

Mauricio Sandoval Cordero, Facultad Latinoamericana de Ciencias Sociales, Costa Rica

Rivera Varela Bertha Leticia, Universidad Abierta a Distancia, México

Tomás Sebastián Torres López, Universidad Alberto Hurtado, Chile

\section{CONSEJO ASESOR INTERNACIONAL}

\author{
Alejandro Mejía Tarazona \\ Facultad Latinoamericana de Ciencias Sociales, Ecuador \\ Alexander Luna Nieto \\ Fundación Universitaria de Popayán, Colombia \\ Celeste De Marco \\ CONICET/Universidad Nacional de Quilmes-CEAR, Argentina \\ Christian Andres Quinteros Flores \\ Universidad de Chile, Chile \\ Daniel Orizaga Doguim \\ Centro de Investigaciones Multidisciplinarias-UAQ, México \\ Francisco Javier Jover Mart \\ Universidad de Castilla-La Mancha, España
}

Gaya Makaran

Centro de Investigaciones sobre América Latina y el Caribe CIALC, UNAM, México

Jaime Araujo Frias

Universidad Nacional de San Agustín, Perú

João Luis Binde

Universidade Federal De Pernambuco, Brasil

Luisina Castelli Rodríguez

Universidad de la República, Uruguay

Mariana Jesica Lerchund

Universidad Nacional de Río Cuarto, Argentina

Marina Acosta

Universidad de Buenos Aires, Argentina

Noelia Marina Cortinas

Universidad de Buenos Aires, Argentin

Paulo Alves Pereira Júnior

Universidad Estatal Paulista, Brasil

Sergio Monroy Isaza

Universidad de Ibagué, Colombia

Suyai Malen Garcia Gualda

IPEHCS-CONICET-UNCO, Argentin 


\title{
Territorio, trabajo y políticas culturales: una articulación necesaria en Uruguay
}

\author{
Territory, work and cultural policies: a necessary link in Uruguay
}

Susana Dominzain

Universidad de la República - Uruguay

susanadominzian@gmail.com

\section{RESUMEN}

Este artículo presenta resultados primarios de una investigación que ha sido financiado por la Universidad de la República, 20015-2019. Se han monitoreado la políticas culturales producidas en Uruguay creadas a partir del año 2007, especialmente aquellas que resultan innovadoras y se diferencian de las tradicionales. Así como también aquellas que dan lugar a una nueva institucionalidad donde lo global no parece ser el parámetro a seguir sino más bien el rescate de lo local, nacional y regional. En esta oportunidad las políticas analizadas son Centros MEC, Usinas Culturales y Estatuto del Artista. Los resultados alcanzados nos muestran un país que en términos culturales es heterogéneo, y complejo. A través de ellas se ha podido apreciar una interesante articulación entre el territorio, el trabajo artístico y las políticas culturales. Desde el Estado se han propuesto metas ambiciosas y de envergadura, sin embargo la percepción de la población destinataria dista de ser la esperada. Los efectos de estas políticas son diversos y dejan entrever que aún falta mucho recorrido.

Palabras claves: Territorio; trabajo; política culturales; Uruguay; monitoreo

\section{ABSTRACT}

This article presents primary results of an research that has been funded by the University of the Republic, in Uruguay during 20015-2019. The cultural policies produced in Uruguay created since 2007 have been monitored, especially those that are innovative and differ from traditional ones. As well as those that give rise to a new institutionality where the global does not seem to be the parameter to be followed but rather the rescue of the local, national, and regional. This time the policies analyzed are from the MEC Centers, Cultural Plants and the Artist's Statute. The results achieved show us a country that in cultural terms is heterogeneous and complex. Through them an interesting articulation between the territory, artistic work and cultural policies has been appreciated. Ambitious and far-reaching goals have been proposed from the State, however, the perception of the target population is far from expected. The effects of these policies are diverse and suggest that there is still a long way to go.

Keywords: Territory; work; cultural policy; Uruguay; monitoring 


\section{Introducción}

Sea bajo el discurso de derechos, como ámbito de intervención de la sociedad civil, como mercado o terreno de investigación, actualmente la cultura, constituye un recurso más que valioso, ineludible (Nivón, E, 2013; Yúdice, G, 2002). Durante décadas las fuertes definiciones que legitimaron a la "alta" cultura como "la" cultura en oposición a la cultura popular van siendo sustituidas en Uruguay por el reconocimiento hacia la cultura como un todo polisémico y controvertido. Para que ocurriera este tránsito fue preciso diseñar mecanismos de intervención como lo son las políticas culturales.

La histórica marginalidad de diversidad de prácticas y universos de sentido que están más allá de las expresiones hegemónicas se ha revertido, dando paso a un mayor interés no solo de académicos, intelectuales y artistas sino también de los políticos. De esta manera, se comienza a tomar en cuenta el peso y la incidencia que la cultura, bajo sus múltiples expresiones, logra tener en la conformación de una sociedad democrática, inclusiva y diversa, pero también como espacio de poder. Las políticas culturales ya no son solamente motivo de debates doctrinarios en revistas literarias o suplementos periodísticos de fin de semana. (García Canclini, N, 1987, p. 64).

El estudio de los efectos de las políticas públicas, sea en el contexto institucional, en las tramas sociales, como en el plano más concreto de la vida de los sujetos/destinatarios, es en sí misma una instancia valiosa. Los escenarios sociales van mudando con celeridad y se requiere de perspectivas sustentadas empíricamente, que los expliquen y aporten pautas para los pasos siguientes.

En relación a las políticas públicas hacia la cultura, sobre todo en las producidas en los últimos años, se han instalado discursos contundentes que, como nunca antes, aluden a desmontar expresiones de desigualdad y a generar cambios culturales. No son pocas las políticas que se plantean entre sus objetivos principales "generar ciudadanía", "garantizar el acceso a los derechos culturales", "democratizar la cultura", entre otros. El carácter eminentemente social y redistributivo de las políticas culturales del nuevo siglo y su sentido político hacen pertinente colocar la interrogante acerca de cuáles son esos resultados.

¿Qué ha cambiado en Uruguay en los últimos años, específicamente a partir de políticas que se han extendido a lo largo y ancho del país y que dispusieron de importantes recursos? Podemos decir que la respuesta a esta pregunta recién comienza a cobrar sistematicidad. Desde la investigación se intentan realizar aportes en este sentido, pero sabemos que lo acumulado es todavía insuficiente y una de las razones de tal insuficiencia es que son escasos los estudios de carácter independiente. En general, buena parte de la producción la constituyen evaluaciones internas o consultorías cuyos resultados escasamente se dan a conocer, es decir, que no generan interlocución más allá de puntuales ámbitos del Estado.

\section{Una mirada al pasado}

El surgimiento del capitalismo supuso con mayor evidencia los cambios culturales. Hubo que adaptarse no solo a la ideología de una nueva clase burguesa que surgía sino a sus costumbres y tradiciones que daban lugar a una nueva cultura. En Uruguay, la temprana aparición de la Biblioteca Nacional en 1816, el Archivo General de la Nación en 1827 y el Museo Histórico Nacional que se crea en 1838, son expresión de una época y una concepción de la cultura. Una prematura institucionalidad se hace presente en el país. Lo que habilita que algunos autores entiendan que las políticas culturales, en el caso uruguayo, antecedieron al Estado-Nación.

La cultura surge como preservadora y difusora de las diferentes manifestaciones artísticas, Durante el siglo XIX y primeras décadas del siglo XX, prácticas como las de promover la actuación de bandas de música en eventos o festividades al aire libre, constituyeron formas de esparcimiento popular frecuentes que a su vez contribuían a la difusión de distintos tipos y formas de expresión musical. Algo similar ocurrió con el carnaval, la mayor manifestación popular de la época, en la que confluían distintas disciplinas artísticas, y que fue logrando creciente apoyo del gobierno municipal. También las sociedades filarmónicas habían contado con la adhesión creciente de sectores medios y altos de la sociedad desde el siglo XIX. Sin olvidar la continua y amplia contratación de compañías extranjeras, del género lírico o teatral, resaltando la presencia de elencos de zarzuela que promovieron el surgimiento local de dicho género, o solistas de fama internacional como Enrico Caruso, el director Arturo Toscanini o la bailarina Isadora Duncan, por mencionar algunos.

La institucionalidad, y sobre todo la infraestructura cultural construida a lo largo del siglo XIX y la primera mitad del siglo XX -indica Achugar-, apostó (...) a los espacios museísticos, a los grandes teatros, a las orquestas nacionales y similares. La consigna era crear no solamente un aparato burocrático, legal y educativo, sino también estructuras e instituciones que permitieran armar el edificio cultural de nuestras naciones. Unas apostaron al modelo de las grandes colecciones y los grandes museos; otras, a 
la conservación patrimonial; otras, a la red de teatros y estímulos a la monumentalización urbana. En ese proceso participaron no solo los Estados nacionales, sino también empresarios privados y, por supuesto, la Iglesia. Sin embargo, los emprendimiento de un sector de la sociedad -me refiero a los sectores marginales o subordinados - eran ignorados, censurados o, en el mejor de los casos, cooptados. (Achugar, H, 2018, p. 264)

El Estado incentivó además la creación artística, en especial la creación individual, a través de instrumentos como el encargo (la forma clásica de mecenazgo estatal tradicional), o los premios, muchas veces acompañados de erogaciones destinadas al financiamiento de la realización de una obra, la publicación de un libro o la erección de un monumento. La formación artística como objetivo de una política cultural corresponde en los hechos a un segundo momento o segunda fase del proceso de formación de una institucionalidad para las políticas culturales. A diferencia de la actuación de una banda, del encargo de un monumento o de un premio a una obra literaria, la formación artística requiere una inversión sostenida cuyos resultados se verán solo a mediano o largo plazo (De Torres, I. 2015).

En América Latina los cambios y transformaciones producidos desde el Estado hacia la cultura, han ampliado la masa crítica de académicos, gestores y artistas que se ven interpelados y a la vez desafiados a ejercer una mayor crítica. Dialogar con ellas/os en distintas partes del país y en algunos países latinoamericanos, configuran encuadres muy atendibles en función de recabar los variados puntos de vista. Vivimos en la globalización y conlleva a que no podamos prescindir de otras realidades que se asemejan pero a la vez se distancian de lo que viene sucediendo en términos culturales en Uruguay.

La revalorización que experimenta la cultura otorga una mayor centralidad al tema y vuelve necesario un abordaje, que más allá de interpretaciones aproximadas, obliguen a un renovado y necesario interés en la obtención de información que otorgue resultados que admitan su análisis y transformación. Es en este contexto que los estudios sobre consumo cultural adquieren también una mayor relevancia. Para ello se hizo necesario sortear las limitaciones institucionales pero también metodológicas. Se trató de entender que la gente consume en escenarios de escalas diferentes y con lógicas distintas (García Canclini y Moneta, 1999, p. 77). Y que cada trayecto implica dinámicas de localización y deslocalización de enraizamiento y desarraigo cultural (Jesús Martín-Barbero. 1988, p. 98).

En los últimos años la cultura en Uruguay ha experimentado cambios importantes. El interés por parte de las autoridades se ha manifestado con una serie de políticas culturales que contempló diferentes dimensiones. El reconocimiento a los derechos culturales y a la ciudadanía cultural se hizo evidente a través de programas, políticas y medidas que tienen por objetivo atender a los sectores más vulnerables de la sociedad. Así como también el manifiesto interés por parte del Estado hacia los trabajadores de la cultura.

En lo que respecta a la institucionalidad y financiamiento, la Dirección Nacional de Cultura adquiere carácter de unidad ejecutora lo que posibilita que la cultura cuente con presupuesto propio. Ya no más migajas para la cultura, constituye un deseo largamente esperado que se concreta puesto que no podemos hablar de políticas culturales si ignoramos sus soportes económicos (Caetano: 2015, p. 18).

Todos estos logros han requerido de decisiones políticas pero fundamentalmente de la elaboración, planificación y gestión de políticas culturales que dan respuesta a necesidades largamente esperadas. Durante mucho tiempo se atribuyó a las políticas culturales la acción de preservar. La creación de museos, archivos, bibliotecas, fueron expresión de lo que debería atender la cultura. Una manera de eternizar y dar continuidad a una de las formas que el Estado utilizaba en la construcción de una nueva nación.

La cultura colaboró en dar forma a una fuerte hegemonía ante una nueva época emergente que exigía del orden y de la paz en países donde campeaba la violencia. Dar término a la sociedad bárbara, al decir de Barrán y disciplinar al colectivo social se hizo imperioso y en ello la cultura jugó un papel fundamental (Barrán , J. P. 1990, p. 9).

\section{Las políticas desde afuera}

Al menos desde comienzos del siglo XX, podemos advertir la existencia de instancias de negociación y conflicto en torno a recursos materiales y simbólicos entre el Estado y el campo cultural, así como negociación y conflicto entre los actores de los distintos sectores de este campo en su búsqueda de reconocimiento por parte del Estado. En otras palabras, podemos encontrar políticas culturales en tanto políticas públicas. Será en la finalización de la Segunda Guerra Mundial que el interés sobre la cultura se haga más evidente y surjan declaraciones referidos a los derechos ciudadanos. En 1948 la Declaración Universal de Derechos Humanos consagra la afirmación de que "todo ser humano tiene derecho a 
participar libremente en la vida cultural de la comunidad". Sin embargo, es en la Convención Internacional de la UNESCO de 1970 donde su director general René Maheu enuncia directamente el "derecho a la cultura" la responsabilidad que compete a las autoridades de proveer los recursos para hacer efectiva esta participación que parece diluirse en América Latina.

Es así como a partir de los '70 se realiza una serie de conferencias intergubernamentales sobre políticas culturales, en Venecia (1970) y en México (1982). La definición elaborada por UNESCO en los años sesenta sirve para ilustrar este modo de entender la política cultural: "la suma total de los usos, acciones o ausencia de acciones de una sociedad, dirigidas a la satisfacción de ciertas necesidades culturales a través de la óptima utilización de todos los recursos materiales y humanos disponibles a una sociedad determinada en un momento dado" (UNESCO, 1968). Esta definición es resultado de una de las reuniones más antiguas que organizó UNESCO para discutir este tema (diciembre de 1967, Mónaco). A la mesa redonda asistieron responsables de cultura de 24 países (en esa época, los ministerios de cultura eran escasos). La definición debe ser tomada con cuidado, pues la intención del organismo internacional deseaba evitar la imposición de un criterio normativo. De tal modo, en la presentación del documento citado se advierte que la "política cultural" debe ser aprehendido como un cuerpo de principios operacionales, prácticas y procedimientos administrativos y presupuestarios que proveen de una base la acción cultural del Estado. Obviamente no puede existir una política cultural seguida por todos los países; "cada Estado Miembro determina su propia política cultural de acuerdo con los valores culturales, objetivos y elecciones establecidas por el mismo". (UNESCO, 1968)

Esta definición condujo con el tiempo a pensar claramente las políticas culturales como políticas públicas, que las podemos definir, en el caso que nos ocupa, como medidas públicas tomadas en el campo cultural. A partir de estas conferencias/convenciones se logró introducir un cierto sentido común internacional: el crecimiento de los países no puede evaluarse solo por índices económicos; y que el desarrollo cultural, concebido como un avance conjunto de toda la sociedad, requiere de políticas públicas específicas y no puede ser dejado como tarea marginal de elites refinadas o librado a la iniciativa empresarial de grandes consorcios comunicacionales (García Canclini, N, 1987, p. 120).

Asimismo, en los últimos años la propia concepción de cultura cambia, redefiniéndose el diseño de políticas culturales. Las industrias creativas se constituyen crecientemente en objeto de políticas culturales y las tecnologías audiovisuales en preocupación de los gestores en vistas a atender los nuevos patrones de consumo cultural de los ciudadanos.

\section{¿Políticas para qué?}

El auge de políticas culturales de impronta social y redistributiva, la formación y profesionalización de las y los trabajadores del sector es una circunstancia en vías de consolidación. Entre distintas vías de formación cabe resaltar que la oferta académica pública se ha ampliado y, lo que no es menor, alcanza el nivel de especialización.

Esto no solo se debe a la ampliación de la masa crítica en el campo cultural, también trae una cuota aparte en el afianzamiento de algunos grupos de investigación, que consecuentemente han colocado su producción de conocimientos sustentados desde lo empírico. Así, de forma creciente "la cultura" va dejando de ser un exclusivo terreno de reflexión erudita; ahora también tienen voz saberes aplicados, nuevas generaciones de trabajadoras/es culturales con valiosas experiencias e incluso destinatarios.

Nos encontramos en un momento de avidez entre los diversos actores que mantienen vínculos con el terreno de las políticas culturales y de producción de puentes entre artistas, gestores e investigadores.

El cambio en los objetivos y en los instrumentos de intervención de las políticas públicas no siempre comporta el desarrollo de nuevas perspectivas y metodologías para su evaluación. Si bien las políticas culturales han adquirido una función preponderante en el desarrollo territorial, buscando integrar una economía del conocimiento con la cohesión social, la gobernanza y la sostenibilidad, es necesario complementar las perspectivas de evaluación hasta ahora trabajadas solo desde criterios de consumo cultural y/o de externalidades de la cultura (Barbieri, Partal, Merino: 2010, p.110).

En tal sentido, esta investigación se propuso contribuir al análisis de políticas culturales, su valor público y los beneficios producidos para la ciudadanía. Analizar de manera crítica y documentar estas políticas culturales, estudiar los procesos que transcurren en los territorios donde se desarrollan, así como las percepciones y apropiaciones que realizan los sujetos destinatarios. Se interpela, en la medida que se trata, al menos en lo que respecta a Uruguay, de un campo de estudio desatendido.

Vale decir, a partir de los trabajos realizados se ha relevado información acerca de cuáles son las 
políticas culturales existentes (Dominzain, S, et al., p. 2014.) y se ha aproximado a conocer cómo impactan a nivel macro en determinadas dimensiones de lo social; asimismo, estudiar cómo estas políticas llegan a sus destinatarios, qué usos hacen estos de las políticas y si las mismas colman las necesidades de los grupos o poblaciones a las que están dirigidas. En qué contexto de sentido se ubican.

Rosalía Winocur señala la importancia del análisis de las representaciones de los distintos actores que participan en las políticas, en la medida que inciden y condicionan el alcance y la pertinencia de las mismas. "Todo diseño de políticas involucra racionalidades distintas. Entre el diseño de las políticas y la puesta en práctica de las mismas opera un proceso de resignificación constante donde el mismo texto puede interpretarse y desarrollarse de maneras distintas, según sea un burócrata de municipio, un político, un maestro de disciplinas artísticas o un docente de oficios. Y este proceso se complejiza con el tiempo, ya que la interacción creciente con el usuario del programa introduce nuevos elementos que a su vez inciden en la interpretación de la realidad y de los criterios con los que se opera" (Winocur, R, 2010, p. 24).

Existe el convencimiento que documentar las políticas culturales sigue siendo una tarea indispensable para poder hablar de ellas, o sencillamente para evitar la desmemoria de nuestros pueblos. En varios países de América Latina es algo que aún debe ser iniciado. Pero para que tal registro alcance cierta objetividad y valor explicativo, necesita encarnadura, no solo desde los protagonistas o los poderes responsables de las acciones, sino mediante un trabajo de investigación que evalúe las políticas en relación con sus resultados, con la recepción y refuncionalización que tales políticas sufren al llegar a sus destinatarios (García Canclini, N, 198, p. 46).

\section{Los efectos de las políticas}

Entendemos que estudiar los efectos de las intervenciones estatales contribuye a mejorar la racionalidad instrumental de la implementación de las políticas públicas, permitiendo conocer mejor la realidad sobre la que el decisor estatal interviene (comparación y objetivos), instando a la concreción de análisis de la eficacia de la acción gubernamental (Rausell Köster, Pau 2007, p. 18). No se trata de calificar lo realizado por el Estado, sino de aportar elementos que valoren y dimensionen la acción pública a través del impacto que las políticas han tenido en las poblaciones-objetivo y la pertinencia de las mismas.

Partimos del supuesto que las políticas culturales en Uruguay no solo buscan satisfacer necesidades sino abrir oportunidades que faciliten el acceso a la cultura y en reconocer en cada ciudadano un potencial productor de la misma, así como también atender las demandas y necesidades de sus trabajadores, con el fin de contar con una cultura democrática, plural y diversa.

A su vez reconocer como problema a la institucionalidad cultural es una de las formas de iniciar este proceso. De esta manera no solo se dará respuesta a las necesidades que en el ámbito cultural nos plantea el nuevo siglo donde la cultura adolece del suficiente reconocimiento y carece de oportunidades ante una mirada político-jurídica instalando una especie de ceguera que incide en que veamos con claridad la necesidad de transversalidad e interdisciplinariedad que los estudios culturales requieren (Carámbula, G, 2013, p. 169).

De todos modos esto muestra distintos matices a estudiar. Las políticas culturales han llegado para quedarse y ese parece ser hoy el desafío teórico-político a tener en cuenta. Su existencia como instrumento de intervención para promover e incentivar la creatividad y participación ciudadana y la contribución que esto supondría en el desarrollo de nuestros países, trae aparejada la preocupación sobre cómo atendemos estos procesos, no solo desde los gobiernos sino también desde la academia; lo que redunda en que su estudio se plantee como prioritario.

Para ello se realizaron entrevistas en los diferentes departamentos del país indagando en el grado de profundización y reconocimiento, en el conocimiento y uso de estas políticas. El estudio de campo fue complementado con el relevamiento y análisis de documentos y fuentes elaboradas desde el Estado. Se afianzó con talleres realizados con los/as artistas donde fueron escuchadas sus opiniones y alternativas planteadas.

Aplicar estas metodologías permitió conocer no solo dónde sino cómo se aplican las políticas en territorio y qué grado de conocimiento se tiene de lo local en términos geográficos, pero también emocionales y afectivos, es decir, cómo es la idiosincrasia de la gente, cuáles sus deseos y necesidades en términos culturales. En qué medida la política en estudio colma sus demandas y expectativas. Los aportes y opiniones de los destinatarios de las políticas resultaron ser medulares para conocer los efectos provocados en su aplicación y en el uso de las mismas.

Las dificultades que se presentan aluden a los destinatarios que constituyen poblaciones que durante décadas han permanecido al margen del sistema e invisibilizadas desde y por el Estado. Es 
por ello que se comportan de manera confusa y ante la llegada a la localidad de algo nuevo que tiene como ejecutor al Estado los ciudadanos reaccionan de forma esquiva ante la aplicación de una política determinada. La presencia de una oficina del Estado en la localidad confunde y se asocia con el Estado recaudador o benefactor. De tal modo se produce, por ejemplo, ante la instalación de un Centro MEC. Estos centros consisten en una red de espacios del Ministerio de Educación y Cultura -desarrollados en sociedad con gobiernos locales, departamentales y ANTEL, entre otros-, los cuales funcionan como espacios de construcción de ciudadanía y puntos de encuentro entre los vecinos de cada localidad, las intendencias, los municipios, distintas organizaciones sociales y los trabajadores del ministerio. De acuerdo a la información del Ministerio de Educación y Cultura existen 127 desde donde se organizan actividades educativas, culturales, vinculadas a la divulgación de innovaciones científicas y tecnológicas, la alfabetización digital de adultos (a través del Plan Nacional de Alfabetización Digital-PNAD) y a la circulación de bienes y servicios culturales. Esta política de democratización se lleva adelante a través de una práctica descentralizada en todo el país. Para algunos habitantes la presencia estatal indica la asistencia pero no necesariamente se la asocia a lo participativo y menos aún en el ámbito cultural.'

Por su parte las Usinas Culturales tienen una menor cobertura a nivel nacional y dependen de la Dirección Nacional de Cultura. Son centros regionales equipados con sala de audio, estudio de video, centro de edición y producción, sala de música con instrumentos, espacio multiuso para talleres. Su objetivo es promover el potencial creativo a partir del uso de nuevas tecnologías relacionadas con la comunicación y la cultura y estimular la formación y producción cultural promoviendo la inclusión social especialmente de jóvenes. Como señala Deborah Duarte hay tres instancias en la presentación de la concepción de las Usinas. Una es la proveniente del Estado en sus documentos oficiales; otra, parte de los fines de esas políticas, y luego la apropiación y producción de sentido que de ellas hacen las personas que participan (Duarte, D, 2018, p. 45).

Es así como la implementación que venimos estudiando ha contribuido a redefinir los límites y las expresiones del "trabajo cultural" en Uruguay. Es necesario reconocer cómo además en el nuevo siglo el sistema político uruguayo se ha visto transformado a partir de 2010 con la aparición de una nueva figura política de la que poco se sabe y se habla, el alcalde. Un actor político que logra tensionar o favorecer la relaciones de cercanía, dando lugar a una trama política más compleja en el entendido que su elección es independiente y su pertenencia partidaria puede ser la misma que el intendente o no, y a su vez en la localidad donde ejerza su cargo, el partido al cual pertenece esté constituido en minoría o mayoría. Todo lo cual incide en el desarrollo de sus funciones y los apoyos con que cuente. Este nuevo encare de institucionalidad política y que busca mostrar atisbos de descentralización, repercute en las políticas culturales, así dan testimonio los Centros MEC cuyo funcionamiento y continuidad muchas veces se ve condicionado por estos cambios que se han procesado en la política nacional y que al momento de crearlos, no fueron tenidos en cuenta.

Conforme a esos desniveles, se implementan medidas descentralizadoras buscando fortalecer la democracia cultural, sin embargo no siempre se materializa y es preciso procesarlos con el consabido transcurrir del tiempo. Para que la descentralización se sostenga "es necesario el crecimiento y complementariedad de los recursos locales y centrales; contratación o formación de personal capacitado; institucionalización del aparato cultural local y desarrollo de una normatividad adecuada. La lentitud o rapidez con que se realice el esfuerzo descentralizador dependerá del desarrollo adecuado de estos factores" (Nivón, E, 2016, p. 20).

Al unísono, tal proceso de descentralización necesita de una institucionalidad cultural ordenada y con cierta coherencia que en nuestro país no existe. Lo que ha llevado a que prestigiosos intelectuales y jerarcas definan a la institucionalidad como un archipiélago cultural, desordenado y que históricamente ha permanecido fragmentado, lo que da muestra de su debilidad a la hora de sustentar políticas y medidas que necesitan de respaldo esencialmente ministerial (Achugar, H. 2013, p. 169)

Se producen avances, se elaboran políticas pero en lo esencial, no se modifican los problemas estructurales en materia de política pública cultural en Uruguay. Es lo que Carámbula llama la institucionalidad por aluvión, refiriéndose a la acumulación de instituciones sin una lógica sistémica coordinada (Carámbula, G, 2013, p. 295).

Por otro lado, la política cultural no es solo un asunto del Estado, o no debería serlo. A su vez, a pesar de proponérselo con categorías discursivas contundentes la inclusión de las políticas resulta ser

1 Información obtenida de la página web. http://www.centrosmec.gub.uy/innovaportal/v/19627/31/mecweb/ que-es-centros-mec?breadid=null\&3colid=19625 Consultado el 9 de setiembre de 2019. 
insuficiente. Las minorías no son contempladas más allá que en estos últimos años los cambios producidos son reales y han sido concretos pero no resultan suficientes.

Se facilitó el acceso a la cultura pero relativamente y en particular a lo que refiere al consumo. Sin embargo continúa limitado al territorio donde vivimos. Además de la incidencia que logra tener la clase social o el capital cultural en el acceso a la cultura, transcurre en el plano nacional de acuerdo a la región perteneciente, la localidad y el barrio. Igual impronta se produce en Montevideo.

Pese a la descentralización incompleta y a la institucionalidad desordenada y fragmentada se ha podido avanzar, no obstante las desigualdades sociales y culturales persisten, se agudizan visualizándose en los consumos. Muchas veces el Estado incursiona para llenar vacíos o para abrir oportunidades. Si, como señalamos antes, buena parte de las políticas del nuevo siglo denotan un carácter fuertemente social, parece pertinente interrogarnos sobre su alcance, en particular tratándose de "minorías" poco visibles y que por su particularidad interpelan los modelos de política pública tradicional.

El proceso de democratización que experimentó la cultura se ha manifestado en la atención hacia los artistas y creadores estimulando y apoyando su profesionalización en búsqueda de la excelencia. Para ello se apuesta a su formación. Se promueve a través de becas y salidas al exterior de elencos principalmente sostenidos por el Estado. El caso del elenco de ballet del SODRE es un buen ejemplo.

Se incentiva a los jóvenes a que opten por carreras artísticas en su formación académica lo que años atrás era impensable. La creación del bachillerato artístico en Enseñanza Secundaria y Facultad de Bellas Artes a nivel universitario ha permitido que el deseo de ser artista se logre concretar para muchos/ as. Todos estos cambios han contribuido a una mayor expansión cultural en el teatro, la danza, la música, entre otros. Se advierte el incremento de gente joven que aspira a su crecimiento profesional y su inserción en el campo de la cultura, con la expectativa también que se constituya en su fuente de ingresos.

Lo cierto es que los artistas se encuentran en un régimen de multiempleo, son zafrales, en ausencia de un manifiesto régimen legal que se debe aplicar, con un futuro incierto en lo que a seguridad social refiere, que dependerá de criterios aleatorios a la hora de registrarlos. Son estos elementos a tener en cuenta para que la legislación se vaya paulatinamente adaptando a las nuevas realidades de trabajo que plantea para la cultura el proceso de globalización y el desarrollo tecnológico que desafían al colectivo que nos convoca.

La ley del Estatuto del Artista y Oficios Conexos (2008) está en vigencia y se aplica. ¿Qué es lo que entorpece su eficiencia? Por un lado, la ausencia de difusión. Hay un alto porcentaje de artistas que no saben de su existencia y si la conocen no advierten de sus contenidos y los beneficios que les genera, en particular a la danza, el teatro y la música. Tal desconocimiento acarrea que muchos artistas aún no se hayan registrado en el Ministerio de Trabajo. A fin de darle curso, deben presentar una relación de méritos que acredite su formación en el área. Esta información es valiosa pues saber cuántos/as son y conocer sus perfiles permite diseñar políticas públicas que contemplen necesidades y demandas. A la vez estar registrado implica un reconocimiento del Estado y al mismo tiempo marca una significativa diferencia con el artista amateur que tiene trayectoria pero no posee documentación ya que la mayoría de las veces trabaja "en negro".

Sin embargo, mientras acontecen estas transformaciones el conocimiento intelectual y el debate al respecto es apenas emergente. Hay una dimensión sociopolítica sobre este aspecto que no nos podemos permitir desatender y es el hecho de que buena parte de las y los trabajadores de la cultura sean jóvenes tanto en áreas tradicionales, como en aquellas que se han abierto recientemente y están más ligadas a la gestión.

Como se ha encontrado en la presente investigación en curso, uno de los indicadores salientes de los últimos años -y en cierta medida producto de las nuevas políticas implementadas- es la existencia de un amplio número de profesionales y técnicos, trabajadoras/es de la cultura, que reflexionan sobre su tarea y se comprometen con aquellos objetivos políticos. Ellas/os no solo condujeron al Estado a terrenos inexplorados desde la óptica cultural con su labor, sino que ampliaron la masa crítica sobre este campo a partir de su propia experiencia; por tanto, su saber importa y ha de ser considerado. Dialogar con sus protagonistas en distintas partes del país, visibilizar y sistematizar en distintos ámbitos sus puntos de vista, fue medular en esta investigación.

\section{Reflexiones finales}

Luego de lo ya estudiado durante estos últimos cuatro años se ha podido constatar que Uruguay muestra un escenario innovador y complejo en lo que respecta a sus políticas culturales, pero 
con características similares a las iniciativas adoptadas por los países vecinos. La política cultural se ha construido como un terreno transfronterizo, pero orientado a repercutir en las matrices identitarias y de sentido de lo nacional. Es un terreno donde la relación global/local cobra expresiones y tensiones concretas.

Justamente lo novedoso en ejecutar políticas como las estudiadas a partir de 2014 (Centros MECUsinas Culturales y Ley del Artista) es que permiten descubrir un país que confirma su heterogeneidad cultural. Se coincide en que la aplicación y desarrollo de las políticas incide de manera sustancial en el territorio en cuestión, puesto que sin atender a los destinatarios, sus opiniones y aportes es imposible evaluar si a dicha política se la conoce, se la aplica, etcétera. Es lo que nominaríamos una aplicación indiscriminada, sin un estudio de campo previo. Por su propia novedad muchas veces se vuelve intrincada su adaptación porque se desconoce la realidad de cada localidad. Son las denominadas políticas hechas “desde arriba” (Pallares: 2002)

Al mismo tiempo que hay avances en legislación e institucionalidad se presentan dificultades de implementación, zigzagueos en la aplicación de planes y programas, como cambios de rumbo de políticas públicas de carácter nacional, mostrando cierta conflictividad entre actores e instituciones.

Las políticas culturales del siglo XXI en Uruguay no solo buscan satisfacer necesidades. También se han propuesto generar acceso a la cultura, teniendo en cuenta que en cada ciudadano hay un potencial productor e incluso atender las demandas de sus trabajadores. Es así como se da cabida a inéditas iniciativas con el fin de contar con una cultura democrática, plural y diversa.

Se hace prioritario superar visiones anteriores que impiden ver en su totalidad e integralidad los reales problemas de las políticas y su implementación. Miradas pasadas que llevan a "hipertrofiar" al decir de Gonzalo Carámbula, no solo la realidad en términos regionales y/o territoriales sino operativos en el entendido de aquellos instrumentos de los cuales se vale la gestión cultural, para generar cambios de trascendencia.

Independiente de estos logros aún falta mucho por construir. Se aprecian brechas que expresan desigualdades en el acceso y disfrute, pero en sustancia, se percibe la necesidad que el ciudadano conozca sus derechos culturales. A su vez estos derechos sean asumidos y ejercitados, mayores serán las expectativas e inclinación a una búsqueda de protagonismo participando en la dinámica cultural del país. Se trata pues de pensar a la cultura para y por la gente y se apela a que los sujetos adopten una actitud proactiva generando intervenciones, verbalizando demandas y sintiéndose constructores de su propia cultura, aquella que hace al goce, al entretenimiento, incluyendo también valores de solidaridad, tolerancia, diversidad, etcétera.

Ello implica que los destinatarios de las diferentes políticas las hagan suyas, se las apropien y defiendan evitando que ante un cambio de gobierno caigan en desuso, desaparezcan o se produzca el "efecto barrido" que es como suele denominarse en Uruguay: todo se barre debajo de la alfombra. Tal coyuntura parece ser el eterno problema de la cultura en los países de América Latina. La pluricausalidad que enhebra dicha afirmación nos ha planteado el objetivo de un mapa de investigación del proceso, las variables intervinientes - unas visibles, otras soterradas-, y sus consecuencias.

\section{Conflict of interest}

No potential conflict of interest is reported by the author(s).

\section{Funding}

There is no financial assistance in studies from external parties.

\section{Acknowledgements}

$\mathrm{N} / \mathrm{A}$

\section{REFERENCIAS}

Achugar, H. (2009). Espacios de negociación. Apuntes para una nueva institucionalidad en Uruguay, en Institucionalidad Cultural en el Uruguay. Montevideo: Ministerio de Educación y Cultura.

Achugar, H. (2018). Presentes y futuros: inercias y desafíos. Gestión y políticas culturales de cara al futuro en Cuadernos del Claeh, 37(107).

Arocena, F. (2011). Regionalización de la Cultura. Montevideo: Universidad de la República-Dirección Nacional de 
Cultura. Programa Viví Cultura.

Barbieri, N., Partal, A., y Merino, E. (2011). Nuevas políticas, nuevas miradas y metodologías de evaluación. ¿Cómo evaluar el retorno social de las políticas culturales? Papers. Revista de Sociologia, 96(2).

Bauman, Z. (2013) La cultura en el mundo de la modernidad líquida. México: Siglo XXI.

Caetano, G. (2003). Políticas culturales y desarrollo social. Algunas notas para revisar conceptos. Pensar Iberoamérica. Revista de Cultura, (4). Recuperado de https://bit.ly/3d17FrP

Carámbula, G. (2011). La institucionalidad cultural pública como problema. En, F. Arocena (Coord), Regionalización de la Cultura. Montevideo: Universidad de la República-Dirección Nacional de Cultura.

De Torres, I. (2009) Cultura, Diseño Institucional y prácticas democratizantes, en Institucionalidad Cultural en el Uruguay (pp. 179-192). Montevideo: Ministerio de Educación y Cultura.

Dominzain, S et ál. (2012). Primer informe nacional sobre la implementación de la Convención sobre la Protección y promoción de las expresiones culturales, UNESCO 2005. Montevideo, Uruguay: Mastergraf. UDELAR.

García Canclini, N. (1987). Políticas culturales en América Latina. México: Grijalbo.

Martín-Barbero, J. (1998). Euforia tecnológica y malestar en la teoría. Revista. Diálogos de la Comunicación, (20).

Kraniauskas, J. (2015) Políticas Culturales. Acumulación, desarrollo y crítica cultural, México. FLACSO.

Pallares, F. (1988) Las políticas públicas: el sistema político en acción. Revista de Estudios Políticos (Nueva Época), (62).

Nivón Bolán, E. (2013). Las políticas culturales en América Latina en el contexto de la diversidad, en Hegemonía cultural y políticas de la diferencia (pp. 23-45). Buenos Aires: CLACSO. Recuperado de https://bit.ly/2KHDCtb

Nivón Bolán, E. (2006). La política cultural. Temas, problemas y oportunidades. (pp. 19-48). México: CONACULTAFondo Regional para la Cultura y las Artes de la Zona Centro. Recuperado de: https://bit.ly/2KHDCtb

Nivón, E. (sf). Desarrollo y debates actuales de las políticas culturales en México. Consultado el 15 de abril de 2018. Recuperado de https://bit.ly/3bP9UOT

Nivón Bolán, E. (2015). Gestión cultural y teoría de la cultura. México. Gedisa.

Nivón Bolán, E. (2006). La política cultural. Tema, problemas y oportunidades. México: Consejo Nacional para la Cultura y las Artes, Serie Intersecciones.

Rausell Köster, P. (2007). Cultura. Estrategia para el desarrollo local. Madrid: Agencia Española de Cooperación Internacional.

Rausell Kóster, P. (1999). Poder y Cultura: el origen de las políticas culturales [en línea]. En: Políticas y sectores culturales en la Comunidad valenciana: un ensayo sobre las tramas entre economía, cultura y poder. Valencia: Tirant lo Blanch.

SIC SUR. (2012). Los Estados de la Cultura. Estudio sobre la institucionalidad cultural pública de los países del SICSUR. Chile: Consejo Nacional de la Cultura y las Artes.

UNESCO. (1968). Actas de la Conferencia General, 15 a reunión. París, Francia.

UNESCO (2005) ¿Cultura y desarrollo? ¿Desarrollo y cultura? Propuestas para un debate abierto. Madrid. Recuperado de https://bit.ly/2SiorrC

Yúdice, G. (2002). El recurso de la cultura. Usos de la cultura en la era global. Barcelona: Gedisa.

Yúdice, G., y Miller, T. (2004). Política cultural. Barcelona: Gedisa.

Winocur, R. (1996). De las políticas a los barrios: programas culturales y participación popular, Ed. Miño y Dávila, Buenos Aires.

Dominzain, S. (2020). Territorio, trabajo y políticas culturales: una articulación necesaria en Uruguay. Pacha. Revista De Estudios Contemporáneos Del Sur Global, 1(1). Recuperado a partir de http://revistapacha.religacion.com/index. php/about/article/view/7

\section{Author:}

Susana Dominzain

Directora del Centro de estudios Interdisciplinarios Latinoamericanos. Directora del Observatorio Universitario de Políticas Culturales. Facultad de Humanidades y Ciencias de la Educación (Universidad de la República, MontevideoUruguay) 